\title{
SMART assistive systems in power tools - Identification of product requirements in application studies
}

\author{
Jürgen Wilwer ${ }^{1}$, René Germann ${ }^{1}$, Philipp-Tobias Dörner ${ }^{1}$, Sven Matthiesen ${ }^{1 *}$ \\ ${ }^{1}$ IPEK - Institute of product engineering at the Karlsruhe Institute of Technology (KIT) \\ ${ }^{*}$ Corresponding author: \\ Univ.-Prof. Dr-Ing. Sven Matthiesen \\ IPEK - Institut für Produktentwicklung \\ Kaiserstraße 12 (Gebäude 10.23) \\ 76131 Karlsruhe \\ Telefon: 0721 / 60847156 \\ Mail: sven.matthiesen@kit.edu
}

\begin{abstract}
The use of miniaturized sensor and actuator technology makes it possible to develop smart assistive systems in power tools for user support. For this purpose, not only the understanding of the power tool, but also the understanding of the user and the user's behavior are important. Furthermore, the resulting interactions between the user and power tool are indispensable. For the identification of product requirements for smart power tools, this paper presents a methodical approach to analyze the user behavior of non-professionals when assembling a birdhouse. From this analysis, first findings for the need and the added value in the use of smart assistive systems are derived. As a result of this paper first requirements for the development of smart cordless screwdriver can be identified.
\end{abstract}

\section{Keywords}

Power tools, user-centered design, assistive technology, end user studies 


\section{Motivation and state of research}

When using power tools, the user is in a constant flow of force- and information with the technical system. Thus, the user has a significant influence on the working result [1]. In order to increase the quality of the working result independently of the user's performance, more and more assistive systems are built into power tools [2]. Better adaption of the assistive system to the user leads to better achieved work results and higher subjectively perceived suitability of the power tool. In the context of user-centered product development, the design of assistive systems requires knowledge of the extent to which they can and must intervene in the user's work process so that the perceived suitability for use is increased rather than being patronized [3]. For this purpose, it must be known within which applications support by assistive systems is necessary and in the interest of the user. In order to achieve this, relevant applications must be identified and, on the basis of these, requirements for sensors, actuators and information processing must be defined which are necessary for the functional implementation of a suitable assistive system [4]. Preliminary work in the area of use case recognition shows the potential of using machine-learning methods to generate first classification models for power tool applications [5].

There are already several patent specifications describing the idea of using sensors within a hand tool to realize an electronic depth stop and to use this data to control at least one operating parameter $[6,7]$. It is known from the state of the art that the operating parameters and the position of power tools can be recorded and logged [8, 9]. These networking systems, designed for the professional sector, enable the user to record operating and geo-data from several power tools via a data logger integrated in the battery and to manage this data via a tablet or smartphone. The Bosch connectivity platform [10] follows a similar approach in the professional sector. In addition to recording data and exchanging it with the smartphone, the approach developed by Bosch makes it possible to set operating parameters, such as rotation speeds, via the smartphone. Furthermore, first market-ready solutions for intelligent control systems for power tools are available. For the milling of wooden boards the product Shaper@ [11] from Shaper Origin is available. The Shaper is a hand-guided wood milling machine that detects and automatically compensates for positioning errors in the device guide. It emerges from these developments those intelligent systems in power tools are needed and wanted by society. However, the problem is that they do not take the user into account enough or, as is the case with the Shaper, can only focus on one application.

By adapting the product to the user and the application, overall productivity can be increased and the subjectively perceived application suitability can be positively influenced, leading to a better overall assessment of the products suitability of usage [12,13]. The smart products from the state of the art already offer first approaches to support the user, but still the user and the user's behaviour is far too little considered. With the exception of the Shaper@, the presented support systems only map a control and a measurement of the operating parameters, but there is no possibility yet to use this data for an active control of the power tool depending on the user's usage. Therefore, the control of the smart Power-Tool requires the same level of previous knowledge for the respective application for which it is to be used. A cordless screwdriver can be mentioned as an example system. Here, a suitable setting of speed and torque limitation can influence both the quality of the work result and the efficiency in the execution of the work. Especially for non-professional users, this presupposed knowledge can be missing, so that the smart systems from the state of the art add little value to this group of users. As soon as it becomes possible to sufficiently consider the user in relation to the application, the potential for increasing productivity and comfort in the application exists. Therefore, the aim of this paper is to investigate the need for assistive systems for cordless screwdrivers from the perspective of (DIY) users. Based on the clarified needs, requirements for smart systems, which are adapted to the user, are to be derived. To achieve 
this goal, the behaviour in the use of cordless screwdrivers by non-professional users in a near-reality situation has been analysed.

\section{Materials and method}

The following chapter describes the used power tool, sensor devices and the method for the identification of handling problems.

\subsection{Used power tool}

The experiment was performed with a cordless screwdriver PDC Quaddrive 18/4 from Festool (Figure 1) (Festool $\mathrm{GmbH}$, headquarters: Wendlingen am Neckar, Germany). The cordless screwdriver has a four-speed gearbox and a 14-step torque limiter (12 steps with torque-limit +2 steps without torque-limit). The first gear delivers up to $60 \mathrm{Nm}$ of torque at 0 $400 \mathrm{rpm}$, whereas in the fourth gear speeds of $0-3800 \mathrm{rpm}$ can be reached. The standard drill chuck was used. A bit and drill set, as well as a hole saw to fullfill the experiments tasks were provided. The cordless screwdriver has an additional impact drilling function, which was not used in this experiment.

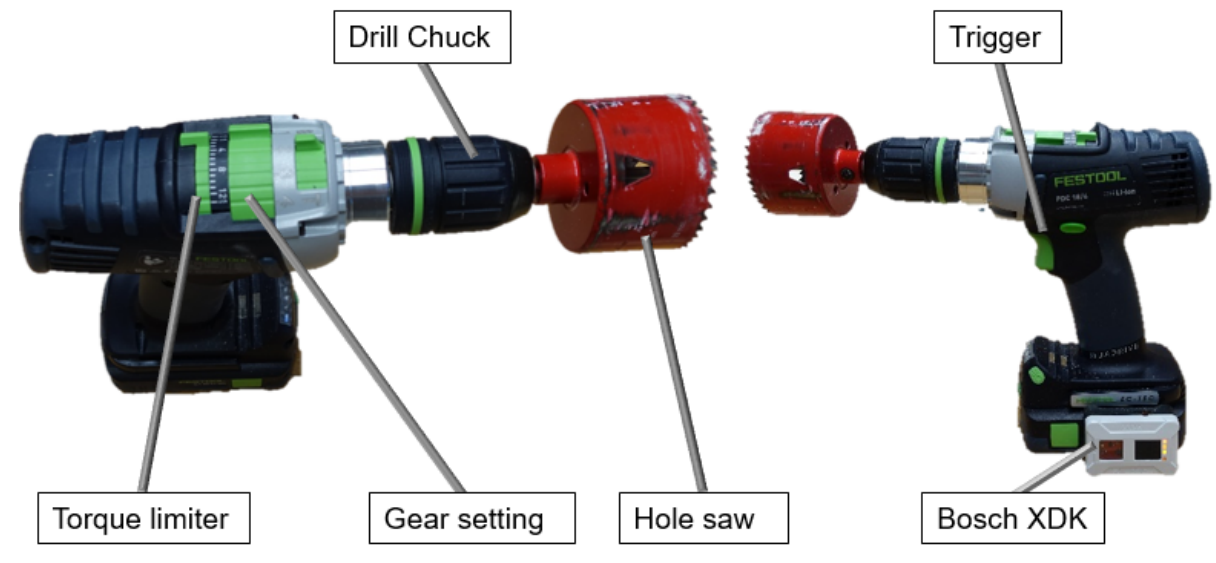

Figure 1: Festool PDC Quaddrive 18/4 with standard drill chuck, mounted hole saw and attached Bosch XDK

\subsection{Sensor devices}

During the experiment, the subjects were observed with four tripod cameras and their ego perspective using eye-tracking glasses. It was decided to use eye-tracking goggles because they can capture the ego perspective better than a forehead camera, since the position of the eye-tracking goggles camera is at eye level of the subject. This perspective was of particular importance for the evaluation of the videos, as it showed which settings were made on the cordless screwdriver. Furthermore, the path of vision data can be used in further research. A Bosch XDK (Cross-Domain Development Kit) [14] was used to generate user-specific data during the use of the cordless screwdriver. The results of the data evaluation of the Bosch XDK represent a part of further research and are not considered in this paper.

\subsection{Methods}

Sixteen subjects took part in this experiment. All subjects were students of the Karlsruhe Institute of Technology (KIT) at the age of 18-26 years.

At the beginning of the experiment, the subjects were provided a paper based multiplechoice questionnaire, the content of which was to record already collected experiences in handling cordless screwdrivers. It was recorded how often each of the subjects works with 
cordless screwdrivers, whether a cordless screwdriver is in their own possession and which activities have already been carried out using cordless screwdrivers. This part of the experiment was designed to ensure that the subjects were explicitly non-professionals.

To analyse and identify relevant procedures and problems in the non-professional user's handling of cordless screwdrivers, an application study was carried out, within which the subjects performed a predefined scenario. The subjects received a systematic video instruction for the assembly of a birdhouse, which had the ambition to cover as many different use cases as possible. The design of the scenario was planned in such a way that the subject had to actively deal with the operating elements of the cordless screwdriver. The use cases include the use of a hole saw, flush screwing and drilling holes with a given drilling depth of $25 \mathrm{~mm}$ for each hole. Each work step also had to be carried out in different working positions to reproduce the influence of difficult to access screws and drill hole positions by purposefully limiting the working area for the experiment and the use of assembly devices that could not be moved by the subject.

For the assembly of the birdhouse, assembly jigs were made and already cut parts of the birdhouse, the necessary screws, drills and other tools such as a folding rule, masking tape and a felt pen were provided (Figure 2, Point 7 and 8 ). The subjects were only allowed to stay in a specific work area, which was marked on the floor (Figure 2, Point 5.) This ensured that the birdhouse was assembled by each subject in working positions as similar as possible. At point 1 in Figure 2, the assembly jig for the birdhouse can be seen. The subjects positioned the individual parts of the birdhouse (Figure 2, Point 7 ) in this assembly device. This made it possible for the birdhouse to be easily assembled by a single subject, as the individual parts could be held in a fixed position. At point 3, Figure 2, the jig for using the hole saw is displayed. Here, the front part of the birdhouse could be mounted, so that neither the subject nor the experimental setup could take any damage, while the hole saw was used. The assembly jig at point 6 , Figure 2, was used so that the roof could be positioned in the correct angle before it was screwed together. Masking tape, felt tip pen and folding rule can be seen at point 8 , Figure 2. The Festool PDC 18/4 with the already mounted hole saw can be seen at point 2, Figure 2. For building the birdhouse, wood screws with lengths of $30 \mathrm{~mm}, 35 \mathrm{~mm}$ and $40 \mathrm{~mm}$ were used. All screws had a diameter of $4 \mathrm{~mm}$.

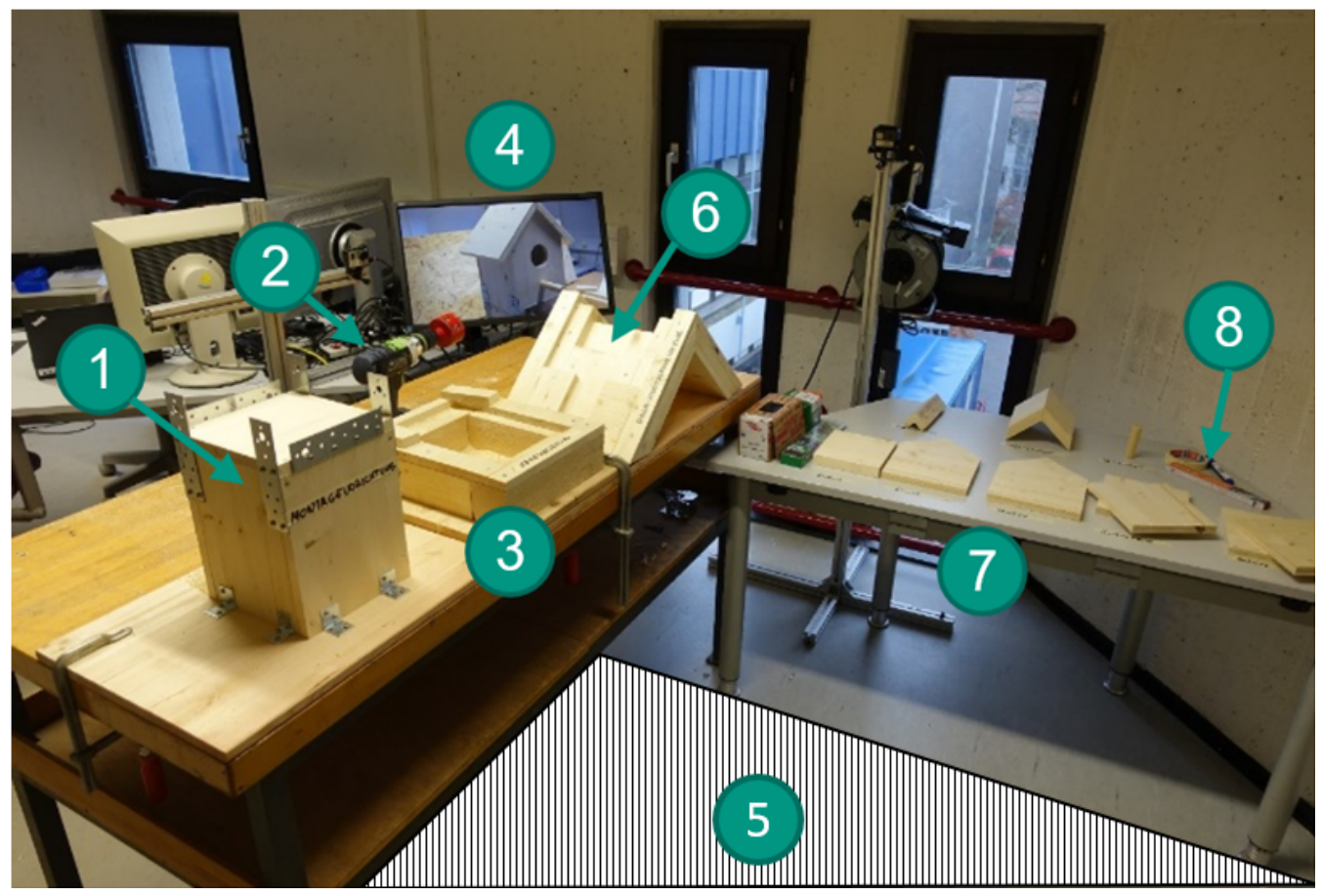

Figure 2: Initial experiment setup, as the subjects encountered at the beginning of the experiment 
In the last step of the study, the subjects were asked in a final questioning to evaluate their work results according to their own perception and the difficulty of fulfilling the task depending on the working position. The questioning started with an open interview recorded in sound and video and was completed with a paper questionnaire.

\subsection{Experimental procedure}

The subjects were observed while assembling the birdhouse. First, a video of the complete assembly of the birdhouse was shown, so that the test persons got a general overview of the individual work steps. The video was played again step by step during the assembly. The video did not show the use of the cordless screwdriver, the selection of the operating parameters suitable for the application, nor the procedure for solving the problem.

In the first step of the assembly the subjects were confronted with the possible operating parameters of the cordless screwdriver by using a hole saw. For this purpose, the cordless screwdriver was placed in first gear and in the lowest level of torque limitation before the test person entered the room, so that the task could not be fulfilled with these settings. The hole saw was already mounted at the beginning of the study. This was to examine how long it took each subject to bring the cordless screwdriver into a functional state to saw the hole. For this purpose, the subject had to recognize that the torque limitation of the cordless screwdriver was at the lowest level. Furthermore, the cordless screwdriver was in first gear. Although this was not functionally relevant, the task could in principle be solved more efficiently with a higher speed. In the next step, the test persons were instructed to drill holes into the parts of the birdhouse. The drill holes should have a depth of $25 \mathrm{~mm}$. It was pointed out in the video that if the hole is not deep enough, there is a risk that the wood could burst, or that if the hole is too deep, the screws could not be fully tightened in the next step. However, no information was given on how to achieve the required drilling depth. The aim of this task was to observe the procedure for measuring the drilling depth. Subjects had the folding rule, the felt-tip pen and the masking tape at their free disposal. Per subject, 13 drillings had to be made. In the third step the parts of the birdhouse had to be screwed together. The aim of the test persons was to screw in the screws flush with the surface. All applications were carried out using the assembly jigs. In total, 21 screws were screwed in per subject.

Overall it was observed whether the different operating modes of the cordless screwdriver are adapted between the different applications. It was investigated how much time nonprofessional users needed to perform the tasks mentioned above. A distinction was made between the total working time for each task and what percentage of the working time was spent on approaching the work result to be achieved and on corrections. During drilling, the time from the first contact between the drill tip and the workpiece until the drill tip left the hole completely was measured. If the drill had to be reinserted in the hole for corrections, the last exit from the hole was taken as the end of the measurement. For screwing in, the start of the measurement was selected as the point in time when the first contact between screw / workpiece and screw / bit existed. The measurement was stopped as soon as the screw was screwed in and the bit finally left the screw head. Here, too, the condition applied that the measurement was continued if subsequent corrections were necessary. The correction time and the time working in slower working speeds to approach the required work result were measured from the first time there was a change in the trigger position. This was determined both visually and via the audio track of the video analysis. The correction time ends analogously to the total time for the respective task. For the evaluation of the working time per task as well as the time for corrections and the approximation to the work result the average over all drillings / screw-in processes was used. Finally, the questioning on the subject's work results was carried out. 


\section{Results}

\subsection{Results of the questionnaire considering working experience}

Eight out of sixteen subjects stated that they used cordless screwdrivers at least once a month. Four out of sixteen subjects used a cordless screwdriver at least once a week. Four out of sixteen subjects stated that they only used a cordless screwdriver at least once a half year. Furthermore, all subjects stated that they already had experience in woodwork in the context of using a cordless screwdriver. Half of the subjects own a cordless screwdriver at home, the second half stated to have access to a cordless screwdriver if needed (e.g. via parents, friends, ...) None of the subjects is considered a professional user, as cordless screwdrivers are only used privately.

\subsection{Monitoring results}

By using the four tripod cameras and eye-tracking glasses, it was possible to take videos that allowed each step of the work and the settings of the cordless screwdriver to be tracked. Figure 3 shows a still image from an analysis video.

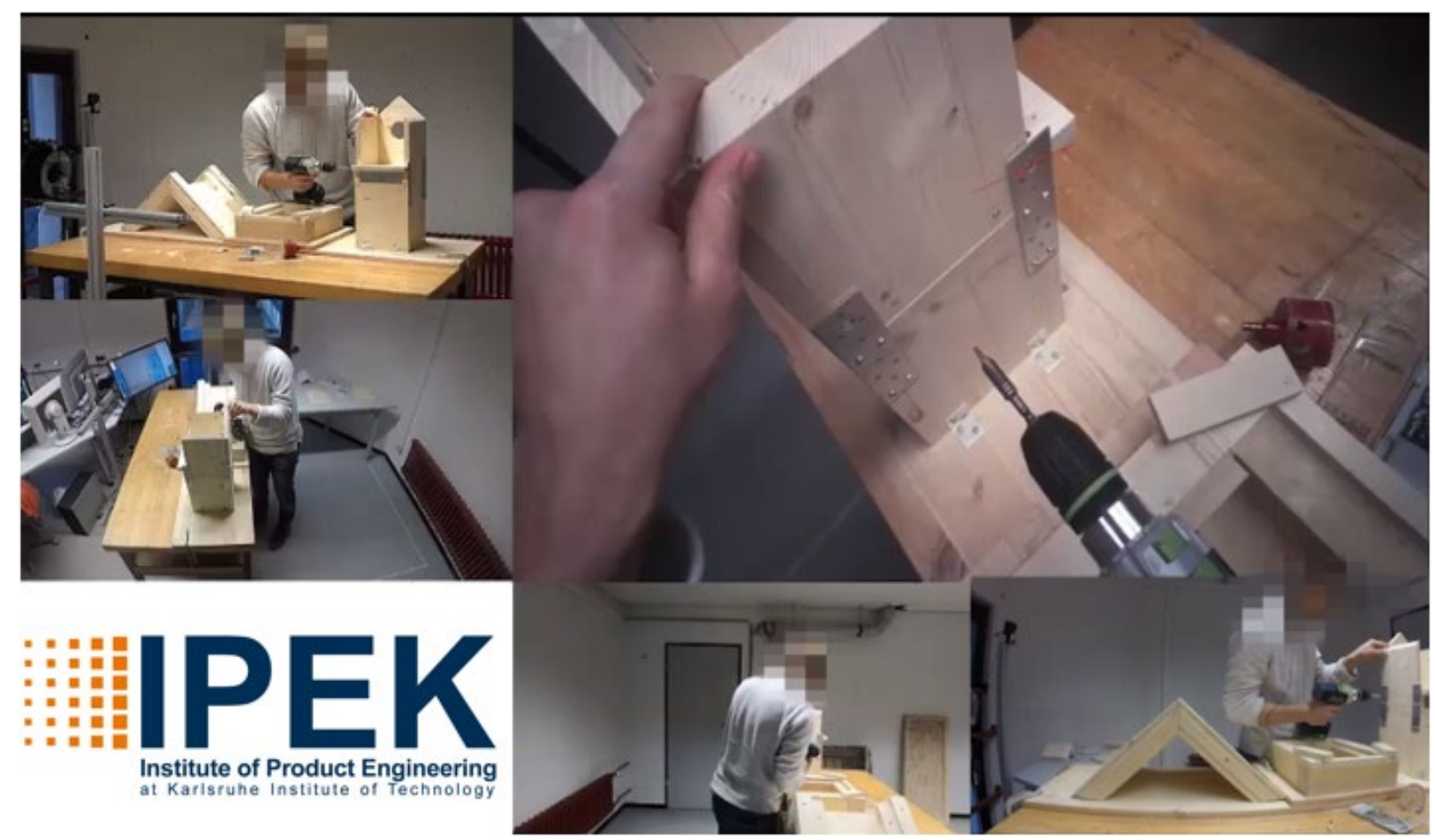

Figure 3: Analysis video from the assembly of the birdhouse

When using the hole saw, fifteen of the subjects followed the same pattern: The cordless screwdriver was used directly without being inspected beforehand. Since a relatively high torque is required from the cordless screwdriver for sawing the hole, the screwdriver gave an acoustic signal to indicate that the torque limiter had intervened. Accordingly, the acoustic signal was chosen as the start time for the commission of the cordless screwdriver and the increase of the torque limit by the user as the end time of the measurement. Figure 4 shows the time needed for each subject to correctly set the torque parameter of the cordless screwdriver for drilling the hole. Only one subject (subject number twelve, Figure 4) increased the torque limit before using the cordless screwdriver, therefore his required time for commissioning the cordless screwdriver was set as zero seconds. 


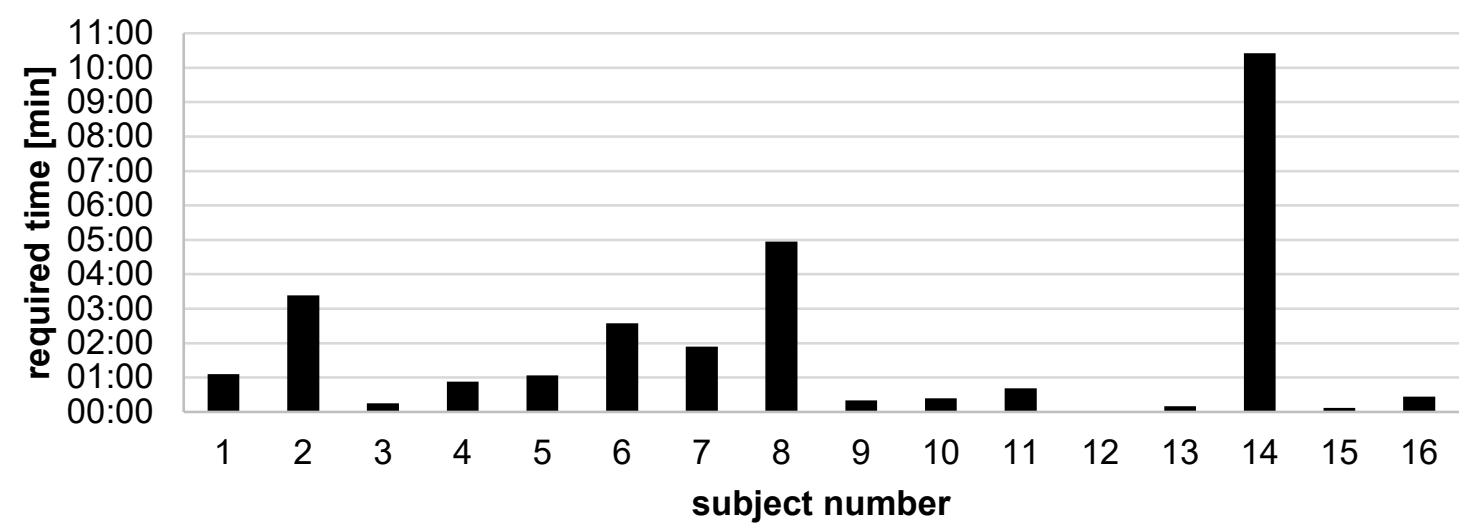

Figure 4: Time for commissioning the cordless screwdriver by each subject

The results of time measurement for drilling and screwing in are shown in Figure 5 and Figure 6 . The black bar represents the total time needed to perform the task and the grey bar represents the time spent approaching the working result on a slower speed and for corrections. For both tasks, the majority of the time was spent approaching the working result with lower working speeds and on corrections (drilling: $61 \%$, screwing in: $79 \%$; both values averaged over all test persons). The evaluation did not consider whether the required drilling or screw depth was actually achieved. Although five of the subjects used the support of the folding rule and masking tape to mark the drilling depth of $25 \mathrm{~mm}$ on the drill, significant differences in the ratio of total working time and correction times with lower working speed can only be observed on the subjects seven and nine (Figure 5). For the subjects four, six and thirteen, no significant differences in the ratio of working and correction times can be observed in comparison to the remaining subjects, who used eye-measurement. The time taken to measure and apply the masking tape was not considered for the total drilling time.

The results show that most of the time needed to perform both tasks is needed for corrections, thus providing the highest potential for improvement.

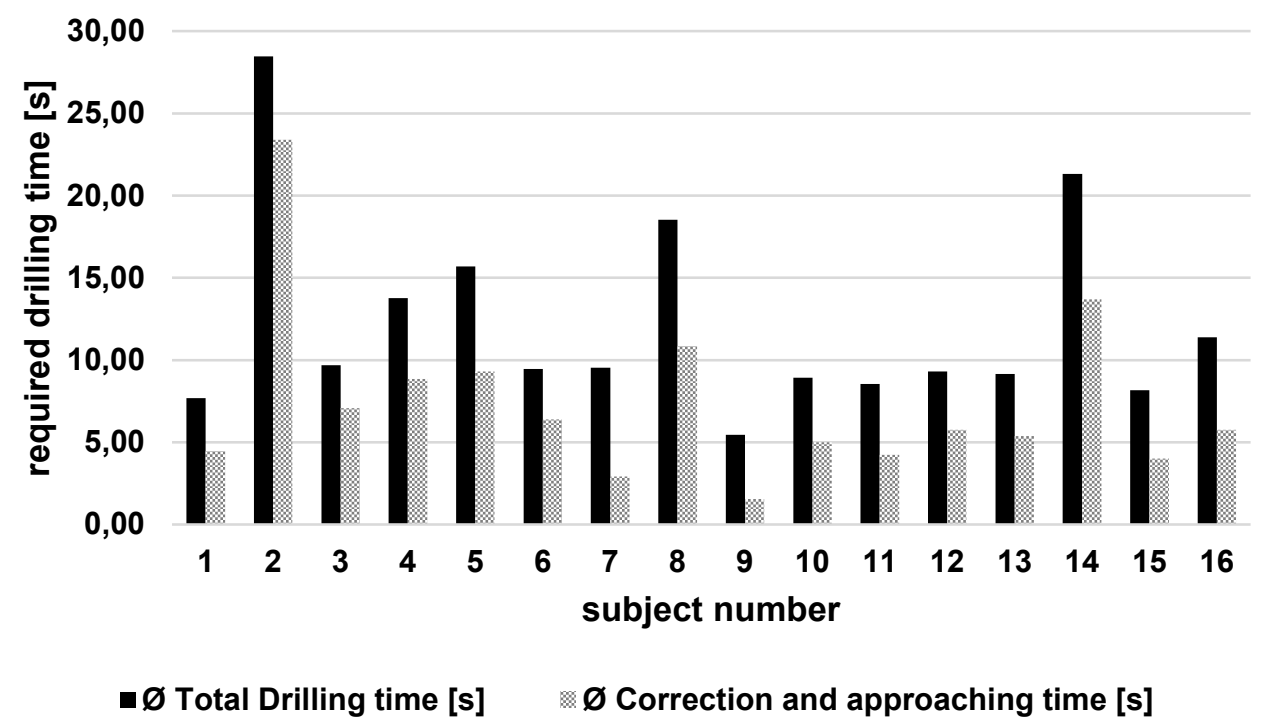

Figure 5: Average time spent for drilling compared to time spent for corrections and approaching the work result 


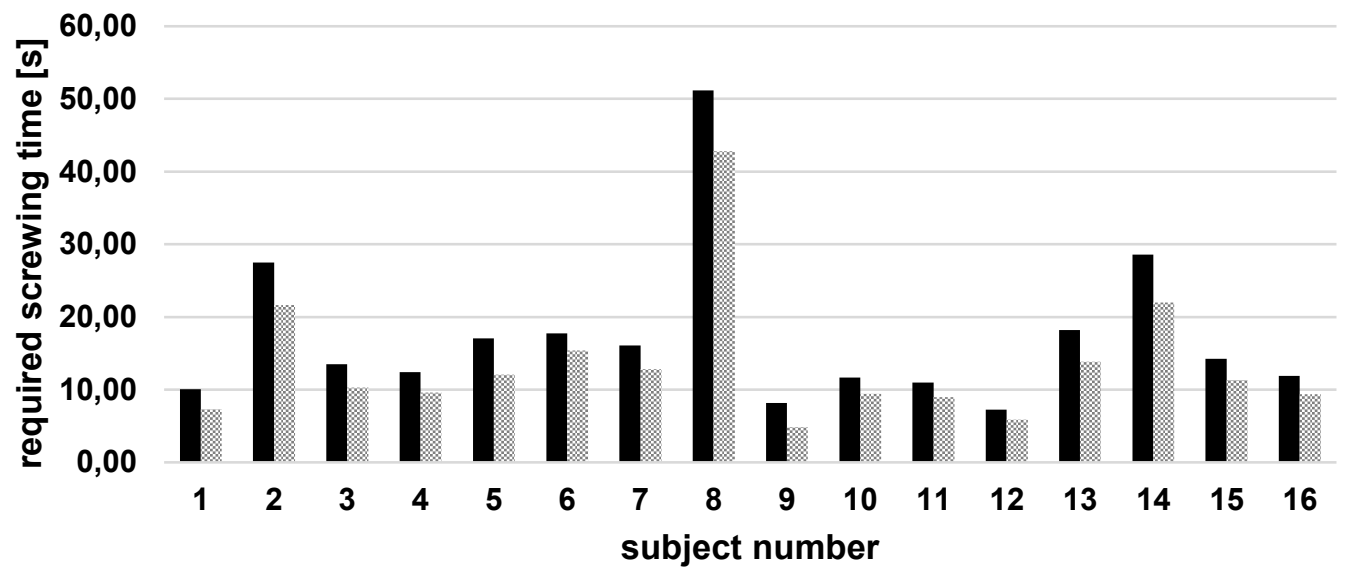

$\varnothing$ Total time screwing in [s] $\varnothing$ Correction and approaching time [s]

Figure 6: Average time spent for screwing in compared to correction time and approaching the work result

Furthermore, the video analysis showed that nine subjects did not make any further adjustments to the parameters of the cordless screwdriver after they had brought the cordless screwdriver into a functional state for the hole saw. Of the seven subjects who actively made adjustments to the cordless screwdriver during the further assembly of the birdhouse, two subjects lowered the torque limit for screwing in, three subjects increased the gear to screw in faster, and two subjects switched between gears and torque limit several times within a single application aimlessly.

\subsection{Results of the questionnaire considering the assessment on the task difficulties}

The subjects stated that the tasks on the front and right side of the birdhouse were easier to perform than on the other sides. However, the subjects also stated that especially the difficult visibility conditions in unwieldy working positions were a decisive factor for the evaluation of the increased difficulty in fulfilling the tasks.

Lastly, the subjects were asked to describe desired improvements for cordless screwdrivers, which are listed in Table 1. Fourteen out of sixteen subjects asked for an automated adjustment of the gear and torque limitation and twelve out of sixteen asked for the automatic shutdown when a previously defined depth is reached. Also, an assistance for the vertical alignment of the cordless screwdriver to the substrate was wished for. Only six of the subjects wanted a function in which the cordless screwdriver automatically switches off when the screw reaches the desired flush position. Furthermore, twelve out of sixteen subjects expressed the wish to receive support for alignment of the cordless screwdriver relatively to a surface, to drill holes at a defined angle, for example.

Table 1: Requested future features by the subjects after the study

\begin{tabular}{|c|c|c|c|c|c|}
\hline Wishes & $\begin{array}{l}\text { Automatic } \\
\text { adjustment } \\
\text { of gear and } \\
\text { torque } \\
\text { limitation to } \\
\text { suit the } \\
\text { application }\end{array}$ & $\begin{array}{l}\text { Automatic } \\
\text { switch-off of } \\
\text { the cordless } \\
\text { screwdriver } \\
\text { when the } \\
\text { screws are } \\
\text { flush }\end{array}$ & $\begin{array}{l}\text { Automatic } \\
\text { shutdown } \\
\text { when a } \\
\text { previously } \\
\text { defined } \\
\text { depth is } \\
\text { reached }\end{array}$ & $\begin{array}{l}\text { Display of } \\
\text { positions for } \\
\text { screws and } \\
\text { drill holes by } \\
\text { e.g. laser }\end{array}$ & $\begin{array}{l}\text { Support for } \\
\text { the vertical } \\
\text { alignment of } \\
\text { the cordless } \\
\text { screwdriver } \\
\text { to a surface }\end{array}$ \\
\hline $\begin{array}{c}\text { Number of } \\
\text { nominations }\end{array}$ & $14 / 16$ & $6 / 16$ & $12 / 16$ & $4 / 16$ & $12 / 16$ \\
\hline
\end{tabular}




\section{Discussion}

It is apparent that subjects who needed a lot of time to start up the cordless screwdriver (Figure 4) had to spend a lot of time to carry out the tasks for assembling (Figure 5 and Figure 6) the birdhouse. This is particularly evident in subjects two, eight and fourteen. This leads to the hypothesis that a lack of knowledge about the adjustment possibilities of a cordless screwdriver can lead to it being used in less than optimal operating conditions. Since the cordless screwdriver was not changed in its operating state after the use of the hole saw by nine subjects, it can be assumed that the subjects required a high correction time during screwing in since for example, the speed of $3800 \mathrm{rpm}$ and / or the renunciation of using the torque limiter with the comparatively short screw lengths led to the situation, that a screw was quickly screwed in too deeply by individual subjects. This required a correspondingly slow approach to the flush condition, which had to be achieved by frequently changing the trigger position. Another factor that can affect the time required to complete the tasks is the respective working position. Especially in working positions, where visibility is also impaired, for most subjects it was difficult to achieve the required work result.

The findings derived from the observation of the experiment largely coincide with the subjects' wishes for a smart cordless screwdriver. However, for the function of the flush screwin only six subjects wished for an assistive function. It can be assumed that the low mentions for a support for flush screwing result from the fact that the focus of the experiment was not to assemble the birdhouse as quickly as possible. Thus, it is possible that the subjects did not actively perceive that almost $80 \%$ of the time for screwing in was spent on approaching a working result due to inefficient adjustments of the trigger position and, if necessary, having to make corrections. This assumption also suggests that the focus was less on time when drilling, but much more on the procedure for estimating the drilling depth, which, as already described, was not familiar to many subjects.

\section{Conclusion and future work}

By observing non-professional users during the assembly of the birdhouse and the following questioning about the problems that occurred during the use of the cordless screwdriver, it was possible to derive main findings for the potentials and benefits in the use of smart assistive systems in cordless screwdrivers:

- The development of an electronic depth stop can increase both the efficiency and the quality of individual tasks. This is especially true for working positions where the user is influenced by poor visibility or uncomfortable postures, for example. Furthermore, the steps of repeatedly adjusting the power tool are omitted when drilling holes of different depths and could possibly be replaced with a faster, more intuitive alternative electronic system.

- Both non-professional and professional users could benefit from the increase in efficiency in the application cases "flush screwing in" and "achieving a defined drill hole depth".

- The use of assistance systems offers the possibility to positively influence the subjectively perceived application suitability of a power tool.

- By using suitable sensor technology, several functions can be implemented that use the same sensor data. As an example, the electronic depth stop could simultaneously provide information about the orientation of the cordless screwdriver relative to the surface.

These main findings result in a larger research project, which raises the question, which requirements for an assistive system in the power tool segment apply. In order to positively 
affect the subjectively perceived suitability of usage of the power tool, relevant factors for the overall assessment are to be identified. After that, their influence on the overall user's assessment of application suitability are to be evaluated. $[15,16]$ In order to develop a control system which is perceived as positive as possible, appropriate investigations must be carried out. The aim of the overall work is to compare the subjectively perceived suitability of usage between a smart and a conventional cordless screwdriver, taking possible disturbance variables from previous usability research into account. This procedure should be carried out on a cordless screwdriver in order to methodically extend this knowledge to other power tools.

\section{Acknowledgments}

This research was supported by the Federal Ministry of Education and Research (Bundesministerium für Bildung und Forschung, BMBF), project number $16 S V 8280$.

\section{Literature}

[1] Schmidt, S.; Hölz, K.; Matthiesen, S.: Use case detailing levels - Anwendungsfallmodellierung zur Unterstützung der Entwicklung handgehaltener Geräte. In: 26. DfX-Symposium, Herrsching, München, 2015.

[2] Schoop E, Nguyen M, Lim D, Savage V, Follmer S and Hartmann: Drill Sergeant Proceedings of the 2016 $\mathrm{CHI}$ Conference Extended Abstracts on Human Factors in Computing Systems - CHI EA '16 the $2016 \mathrm{CHI}$ Conference Extended Abstracts (San Jose, California, USA, 07.05.2016 - 12.05.2016) ed J Kaye et al (New York, New York, USA: ACM Press) pp 1607-14.

[3] Abras C, Maloney-Krichmar D, Preece J.: User-Centered Design. In: Bainbridge W, editor. Encyclopedia of Human-Computer Interaction. Sage; Thousand Oaks, 2004.

[4] Isermann, R.: Mechatronic systems_-Innovative products with embedded control In: Control Engineering Practice 16, 2008 pp 14-29.

[5] Dörr, M. et al: Recognizing Product Application based on Integrated Consumer Grade Sensors: A Case Study with Handheld Power Tools. In: 29th CIRP Design 2019 (CIRP Design 2019), pp 798-803. DOI: 10.1016/j.procir.2019.04.317

[6] Robert Bosch GmbH, EP 1945410 B1, 16.03.2011, page 2, line 37 - 45.

[7] Sears Brands, LLC, Hoffman Estates, IL 60179 (US), EP 3162479 B1, 27.10.2016 page 11 line 1 - 24.

[8] https://www.dewalt.com/jobsite-solutions/tool-connect (last access date: 19.06.2020).

[9] https://de.milwaukeetool.eu/header/news-media/press-releases/2017/one-key-\%E2\%80\%93-der-schlusselzur-digitalen-baustelle/ (last access date: 19.06.2020).

[10] https://www.bosch-professional.com/static/specials/upgrade/gb/en/index.html (last access date: 19.06.2020)

[11] https://shapertools.com/ (last access date: 19.06.2020).

[12] Vink, P.; Koningsveld, E. A. P.; Molenbroek, J. F. (2006): Positive outcomes of participatory ergonomics in terms of greater comfort and higher productivity. In: Applied Ergonomics 37 (4), S. 537-546. DOI: 10.1016/j.apergo.2006.04.012.

[13] Vedder, Joachim; Carey, Eilís (2005): A multi-level systems approach for the development of tools, equipment and work processes for the construction industry. In: Applied Ergonomics 36 (4), S. 471-480. DOI: 10.1016/j.apergo.2005.01.004

[14] Bosch Connected Devices and Solutions $\mathrm{GmbH}$, 2017. Cross-Domain Development Kit | XDK: Start your Sensor X-perience. https://xdk.boschconnectivity.com/documents/37728/87798/XDK_Node_110_combined_Datasheet.pdf/. (last access date: 19.06.2020).

[15] Germann, R. Jahnke, B., Matthiesen, S.: Objective usability evaluation of drywall screwdriver under the consideration of the user experience In: Applied Ergonomics Volume 75, pages 170 - 177, 2019 DOI: 10.1016/j.apergo.2018.10.001

[16] Germann, R. Ribadeneira, E. A., Matthiesen, S.: Objective Evaluation of Usability Aspects for the Development of Handle Geometries In: 29th CIRP Design 2019, pages 502 - 513, 2019, DOI: 10.1016/j.procir.2019.03.295 